\title{
Quadratic reciprocity and Riemann's non-differentiable function
}

\author{
Kalyan Chakraborty ${ }^{*}$, Shigeru Kanemitsu ${ }^{2}$ and Li Hai Long ${ }^{3}$
}

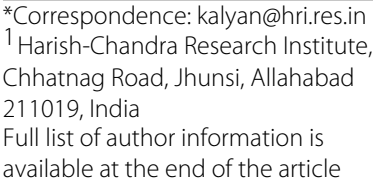

\begin{abstract}
Riemann's non-differentiable function and Gauss's quadratic reciprocity law have attracted the attention of many researchers. Here we provide a combined proof of both the facts. In (Proc. Int. Conf.-NT 1;107-116, 2004) Murty and Pacelli gave an instructive proof of the quadratic reciprocity via the theta-transformation formula and Gerver (Amer. J. Math. 92;33-55, 1970) was the first to give a proof of differentiability/nondifferentiabilty of Riemnan's function. We use an integrated form of the theta function and the advantage of that is that while the theta-function $\Theta(\tau)$ is a dweller in the upper-half plane, its integrated form $F(z)$ is a dweller in the extended upper half-plane including the real line, thus making it possible to consider the behavior under the increment of the real variable, where the integration is along the horizontal line.
\end{abstract}

2010 Mathematics Subject Classification: Primary: 11A15, Secondary: 11F27

Keywords: Quadratic reciprocity; Theta-transformation; Non-differentiable function

\section{Introduction}

Riemann asserted that the function (according to Weierstrass),

$$
f(x)=\sum_{n=1}^{\infty} \frac{\sin n^{2} x}{n^{2}}
$$

is nowhere differentiable. Weierstrass in 1872 tried to prove this assertion but couldn't and instead constructed his own example of a continuous nowhere differentiable function which was of the form

$$
\sum_{n=0}^{\infty} \cos \left(b^{n} \pi x\right)
$$

where $0<a<1$ and $b$ is a positive integer satisfying

$$
a b>1+3 / 2 \pi .
$$

G.H. Hardy [7] proved that Weierstrass's function has no derivative at points of the form $\psi \pi$ where $\psi$ is either irrational or rational of the form $2 A /(4 B+1)$ or $(2 A+1) /(2 B+2)$. Much later in 1970, Gerver [4] disproved Riemann's assertion by showing that his function is differentiable at any point of the form $\psi \pi$ where $\psi$ is of the form $(2 A+1) /(2 B+1)$ with derivative equal to $-1 / 2$. A few years later in 1972, Arthur used Poisson's summation formula and properties of Gauss sums to deduce Gerver's result and there by establishing a link between Riemann's function and quadratic reciprocity (via Gauss sums). The reader

(c) 2015 Chakraborty et al. This is an Open Access article distributed under the terms of the Creative Commons Attribution License (http://creativecommons.org/licenses/by/4.0), which permits unrestricted use, distribution, and reproduction in any medium, provided the original work is properly credited. 
is also refereed to two excellent expositions of Riemann's function by E. Neuenschwander [15] and that of S.L. Segal [16]. This problem was explored by many other authors and among them a few references could be $[5-7,11]$ and [9].

We observe that Riemann's function $f(x)$ is really an integrated form of the classical $\theta$ function. Then we make the link to quadratic reciprocity from an exposition of Murty and Pacelli [14] who (following Hecke) showed that the transformation law for the theta function can be used to derive the law of quadratic reciprocity. The goal of the present paper is to combine these two ideas so as to derive both, the differentiability of $f$ at certain points and the law of quadratic reciprocity.

Our interest in Riemann's everywhere non-differentiable function arose from the following identity (Davenport-Chowla identity):

$$
\sum_{n=1}^{\infty} \frac{\lambda(n)}{n} \psi(n x)=-\frac{1}{\pi} \sum_{n=1}^{\infty} \frac{\sin 2 \pi n^{2} x}{n^{2}} .
$$

Here as usual

$$
\lambda(n)=(-1)^{\Omega(n)}
$$

with $\Omega(n)$ denoting the total number of distinct prime factors of $n$. Also,

$$
\psi(x)=-\frac{1}{\pi} \sum_{n=1}^{\infty} \frac{\sin 2 \pi n x}{n}
$$

is the saw-tooth Fourier series, i.e., it is the Fourier series expansion of the so-called 'sawtooth' function:

$$
f(x)=\left\{\begin{array}{l}
1 / 2(\pi-x), \text { if } 0<x \leq 2 \pi \\
f(x+2 \pi), \text { otherwise }
\end{array}\right.
$$

On one hand in (1) there appears the Liouville function which is a prime numbertheoretic entity and on the other hand, Riemann's example of a nowhere differentiable function. The integrated identity can be derived from the functional equation only, but to differentiate it, one needs the estimate for the error term for the Liouville function which is as deep as the prime number theorem.

As will be seen later, the right - hand side may be viewed as the imaginary part of the integrated theta-series. It seems that the uniform convergence of the left-hand side and the differentiability of the right-hand side merge as the limiting behavior of a sort of modular function and the Riemann zeta-function.

\section{Riemann's function}

Let $p$ be a natural number and $\mathfrak{z}=h+i \epsilon \in \mathcal{H}$ tending to 0 . We denote the upper half-plane by $\mathcal{H}$. Also let for $z \in \mathcal{H} \cup \mathbb{R}$,

$$
F(z)=\sum_{n=1}^{\infty} \frac{e^{\pi i n^{2} z}}{\pi i n^{2}}=\frac{1}{2} \sum_{\substack{n=-\infty \\ n \neq 0}}^{\infty} \frac{e^{\pi i n^{2} z}}{\pi i n^{2}} .
$$

Let us denote by $S(b, a)$ the quadratic Gauss sum defined by

$$
S(b, a)=\sum_{j=0}^{b-1} e^{2 \pi i j^{2} \frac{a}{b}}
$$


for $b$ a natural number. One extends the definition for non-zero integral values $b$ by,

$$
S(b, a)=S(|b|, \operatorname{sgn}(b) a) .
$$

We note that $S(|b|,-a)=\overline{S(|b|, a)}$ and $S(k a, k b)=S(a, b)$.

We begin with the following result:

Theorem 2.1. For any integers $p>0, q$ we have

$$
F\left(\frac{2 q}{p}+\mathfrak{z}\right)-F\left(\frac{2 q}{p}+i \epsilon\right)=S(p, q) \frac{e^{-\pi i / 4}}{p} \sqrt{\mathfrak{z}}-\frac{1}{2} h+O\left(\mathfrak{z}^{2}\right)
$$

where for a non-zero integer $p$, the coefficient is to be understood as $S(|p|, \operatorname{sgn}(p) q)$.

Proof. Let $b$ be an arbitrary real number. One can obtain by using Euler-Maclaurin summation formula as in Lemma 4 in [14] (the resulting integral can be evaluated as in [12] (Page 20-22)):

$$
\sum_{n=-\infty}^{\infty} e^{(b+p n)^{2} i \mathfrak{z}}=\frac{2 \sqrt{\pi}}{p} e^{-\pi i / 4} \sqrt{\mathfrak{z}}+O(\mathfrak{z})
$$

where the branch of $\sqrt{\mathfrak{z}}$ is chosen so that it is positive for $\mathfrak{z}>0$.

We integrate this along the line segment parallel to the real axis, say over $\left[\mathfrak{z}^{\prime}, \mathfrak{z}\right]$ with $\mathfrak{z}-\mathfrak{z}^{\prime}=h$. Now after separating the case $(b, n)=(0,0)$ the integrated form of (3) becomes,

$$
\begin{aligned}
h+ & \sum_{\substack{n=-\infty \\
(n, b) \neq(0,0)}}^{\infty} \frac{e^{(b+p n)^{2} \mathfrak{z}}}{i(b+p n)^{2}}-\sum_{\substack{n=-\infty \\
(n, b) \neq(0,0)}}^{\infty} \frac{e^{(b+p n)^{2} i\left(h^{\prime}+i \epsilon\right)}}{i(b+p n)^{2}} \\
& =\frac{2 \sqrt{\pi}}{p} e^{-\pi i / 4} \sqrt{\mathfrak{z}}+O\left(\mathfrak{z}^{2}\right) .
\end{aligned}
$$

This can be re-written as

$$
T(\mathfrak{z})-T(i \epsilon)=\frac{2 \sqrt{\pi}}{p} e^{-\pi i / 4} \sqrt{\mathfrak{z}}-h(1+o(1))+O\left(\mathfrak{z}^{2}\right) .
$$

Here

$$
T(\mathfrak{z})=T(\mathfrak{z}, b)=\sum_{\substack{n=-\infty \\(n, b) \neq(0,0)}}^{\infty} \frac{e^{(b+p n)^{2} \mathfrak{z}}}{i(b+p n)^{2}} .
$$

Then by the decomposition into residue classes,

$$
\begin{aligned}
F\left(\frac{2 q}{p}+\mathfrak{z}\right) & =\frac{1}{2} \sum_{\substack{n=-\infty \\
n \neq 0}}^{\infty} \frac{e^{\pi i n^{2}\left(\frac{2 q}{p}+\mathfrak{z}\right)}}{\pi i n^{2}} \\
& =\frac{1}{2} \sum_{b=0}^{p-1} e^{2 \pi i b^{2} \frac{q}{p}} \sum_{\substack{n \equiv b \\
(n, b) \neq(\bmod q)}} \frac{e^{\pi i n^{2} \mathfrak{z}}}{\pi i n^{2}} \\
& =\frac{1}{2} \sum_{b=0}^{p-1} e^{2 \pi i b^{2} \frac{q}{p}} \frac{1}{\pi} T(\pi \mathfrak{z}, b) .
\end{aligned}
$$


Now using (5),

$$
\begin{aligned}
F\left(\frac{2 q}{p}+\mathfrak{z}\right) & =\frac{1}{2} \sum_{b=0}^{p-1} e^{2 \pi i b^{2} \frac{q}{p}} \frac{1}{\pi}\left(\frac{2 \sqrt{\pi}}{p} e^{-\pi i / 4} \sqrt{\pi \mathfrak{z}}-\frac{1}{2} h+T(i \epsilon, b)\right)+O\left(\mathfrak{z}^{2}\right) \\
& =\frac{1}{2} S(p, q)\left(\frac{2 \sqrt{\pi}}{p} e^{-\pi i / 4} \sqrt{\mathfrak{z}}-\mathfrak{z}\right)+\frac{1}{2 \pi} \sum_{b=0}^{p-1} e^{-2 \pi i b^{2} \frac{q}{p}} T(i \epsilon, b)+O\left(\mathfrak{z}^{2}\right) \\
& =S(p, q)\left(\frac{1}{p} e^{-\pi i / 4} \sqrt{\mathfrak{z}}-\frac{1}{2} h\right)+F\left(\frac{2 q}{p}+i \epsilon\right)+O\left(\mathfrak{z}^{2}\right) .
\end{aligned}
$$

In (4) the variable can be $\frac{2 q}{p}+\mathfrak{z}$ and $\frac{2 q}{p}+\mathfrak{z}^{\prime}$ and then instead of $h$ we would have $\mathfrak{z}-\mathfrak{z}^{\prime}$. This will be used in deriving (17).

The relation (2) in this form is essentially Theorem 1 of Itatsu [9] and from here nondifferentiability of Riemann's function can be deduced. Indeed, let $\mathfrak{z}=h+i \epsilon$ and let $\epsilon \rightarrow 0+$, in which we have to pay attention to the sign $\operatorname{sgn} h$ of $h$. Then

$$
F\left(\frac{2 q}{p}+h\right)-F\left(\frac{2 q}{p}\right)=S(p, q) \frac{e^{-\pi i / 4 \operatorname{sgn} h}}{p} \sqrt{|h|}-\frac{1}{2} h+O\left(h^{2}\right) .
$$

Hence differentiability follows only in the case $S(p, q)=0$ with differential coefficient $-\frac{1}{2}$. This will be done in the next section appealing to Corollary 3.2. At the same time this is an elaboration of ([14], (47)) (on the right-hand side of which the factor $\sqrt{\pi}$ is to be deleted). Arguing as in [14] using the theta transformation formula, we may deduce the Landsberg-Schaar identity, from which the quadratic reciprocity may be deduced.

Remark 1. We would like to make a few comments on the work of Duistermaat [3].

The equation (3.4) (was already proved by Cauchy [2], pp.157-159) [3] for $r=\frac{q}{p}$ becomes

$$
\begin{aligned}
\mu_{\gamma}(x) & =e^{\frac{\pi}{4} m} p^{-\frac{1}{2}}(x-r)^{-\frac{1}{2}} \\
& =e^{\frac{\pi}{4}} p^{-1} S(2 p, q)(x-r)^{-\frac{1}{2}} .
\end{aligned}
$$

Incorporating this in ([3], (4.1)), we see that it refers to the case $S(2 p, q)$ of our Theorem 2.1. Hence by Corollary 3.2, differentiability of Riemann's function can be read off.

Further on ([3], p. 9, $\ell 7$ from below) the relation (47) in [14] is stated in the form

$$
\Theta\left(\frac{2 q}{2 p}+i \epsilon\right) \sim \frac{1}{p \sqrt{\epsilon}} S(2 p, q), \quad \epsilon \rightarrow 0+.
$$

Thus, we could say that [3] also gives material to deduce the reciprocity law.

\section{Reciprocity law}

Let us denote for $z \in \mathcal{H}$,

$$
\Theta(z)=\sum_{n=-\infty}^{\infty} e^{\pi i n^{2} z}=1+2 \sum_{n=1}^{\infty} e^{\pi i n^{2} z}
$$

and then the classical theta-function for $\operatorname{Re} z>0$ is

$$
\theta(z)=\Theta(i z)=\sum_{n=-\infty}^{\infty} e^{-\pi n^{2} z} .
$$


At this point we note down the theta - transformation formula:

$$
\Theta(z)=e^{\frac{\pi i}{4}} z^{-\frac{1}{2}} \Theta\left(-\frac{1}{z}\right) .
$$

We now prove the reciprocity law.

Theorem 3.1. Let $p \in \mathbb{N}$ and $(0 \neq) q \in \mathbb{Z}$. Then

$$
S(p, q)=e^{\frac{\pi}{4} \operatorname{sgn}(q) i}\left(\frac{p}{2|q|}\right)^{1 / 2} S(4|q|,-\operatorname{sgn}(q) p) .
$$

Proof. Let us first note that $F(z)$ is essentially the integral of $\Theta(z)$ :

$$
\begin{aligned}
\int_{0}^{z} \theta(-i z) \mathrm{d} z & =\int_{0}^{z} \Theta(z) \mathrm{d} z \\
& =z+2\left(\sum_{n=1}^{\infty} \frac{e^{\pi i n^{2} z}}{\pi i n^{2}}-\sum_{n=1}^{\infty} \frac{e^{\pi i n^{2} z}}{\pi i n^{2}}\right) \\
& =z+2(F(z)-F(0)) .
\end{aligned}
$$

In particular, for $z=x+u+i \epsilon \in \mathbb{C}$ (with $\epsilon>0)$ and $u \in(0, h)$, the above relation (10) becomes

$$
\begin{aligned}
\int_{x}^{x+h} \theta(\epsilon-i u) \mathrm{d} u & =\int_{x+i \epsilon}^{x+h+i \epsilon} \Theta(z) \mathrm{d} z \\
& =h+2(F(x+h+i \epsilon)-F(x+i \epsilon)) .
\end{aligned}
$$

The theta-transformation formula (8) with $y>0$ gives

$$
\begin{aligned}
\theta(y-i u) & =e^{\frac{\pi}{4} i} \frac{1}{\sqrt{u+i y}} \theta\left(\frac{i}{u+i y}\right) \\
& =e^{\frac{\pi}{4} i} \frac{1}{\sqrt{u+i y}} \sum_{n=-\infty}^{\infty} e^{\frac{i \pi n^{2}}{u+i y}} .
\end{aligned}
$$

We now make the following change of variable:

$$
\frac{i}{u+i \epsilon}=\frac{i}{x+v+i \epsilon}=\tau+\frac{1}{x} i
$$

i.e.,

$$
\tau=\frac{\epsilon-i v}{x(x+v+i \epsilon)} \sim \frac{\epsilon-i v}{x^{2}} .
$$

Now with this change the integral in (11) becomes

$$
\int_{x}^{x+h} \theta(\epsilon-i u) \mathrm{d} u=-i e^{\pi / 4} \int_{\frac{i}{x+h+i \epsilon}-\frac{i}{x}}^{\frac{i}{x+i \epsilon}-\frac{i}{x}} \frac{1}{\left(\tau+\frac{1}{x} i\right)^{\frac{3}{2}}} \theta\left(\tau+\frac{1}{x} i\right) \mathrm{d} \tau .
$$

The following relation is useful (which is in fact equivalent to (11)) in applying integration by parts:

$$
\int \theta\left(\tau+\frac{i}{x}\right) \mathrm{d} u=\tau-2 i F\left(-\frac{1}{x}+i \tau\right)+C .
$$


Using this, we may evaluate (13) and it becomes

$$
\begin{aligned}
& \int_{\frac{i}{x+h+i \epsilon}-\frac{i}{x}}^{\frac{i}{x+i \epsilon}-\frac{i}{x}} \frac{1}{\left(\tau+\frac{1}{x} i\right)^{\frac{3}{2}}} \theta\left(\tau+\frac{1}{x} i\right) \mathrm{d} \tau \\
= & {\left[\left(\tau+\frac{i}{x}\right)^{3 / 2}\left(\tau-2 i F\left(-\frac{1}{x}+i \tau\right)\right)\right]_{\frac{i}{x+i \epsilon}-\frac{i}{x}}^{\frac{i}{x+h+i \epsilon}-\frac{i}{x}} } \\
= & \left(\frac{x+h+i \epsilon}{i}\right)^{3 / 2}\left(\frac{i}{x+h+i \epsilon}-\frac{i}{x}-2 i F\left(-\frac{1}{x+h+i \epsilon}\right)\right) \\
- & \left(\frac{x+i \epsilon}{i}\right)^{3 / 2}\left(\frac{i}{x+i \epsilon}-\frac{i}{x}-2 i F\left(-\frac{1}{x+i \epsilon}\right)\right)+O(h) .
\end{aligned}
$$

At this point we note that

$$
\frac{-1}{x+h+i \epsilon}=-\frac{1}{x}+\frac{1}{x^{2}}(\mathfrak{z}(1+o(1))) \text {. }
$$

Using (15), the main term in (14) is

$$
\begin{aligned}
& -2 e^{\frac{\pi}{4} i}\left((x+h+i \epsilon)^{3 / 2} F\left(-\frac{1}{x+h+i \epsilon}\right)-(x+i \epsilon)^{3 / 2} F\left(-\frac{1}{x+i \epsilon}\right)\right) \\
= & 2 e^{\frac{\pi}{4} i}(x+i \epsilon)^{3 / 2}\left(F\left(-\frac{1}{x}+\frac{1}{x^{2}} \mathfrak{z}^{\prime}\right)-F\left(-\frac{1}{x}+\frac{1}{x^{2}} \epsilon^{\prime}\right)\right)+O(h),
\end{aligned}
$$

where we have used

$$
\mathfrak{z}^{\prime}=\mathfrak{z}(1+o(1)) \text { and } \epsilon^{\prime}=\epsilon(1+o(1)) .
$$

Now we specify $x=\frac{2 q}{p}$ and apply Theorem 2.1. Under this specification (16) takes the shape

$$
\begin{aligned}
& =2 e^{\frac{\pi}{4} i}\left(\frac{2 q}{p}+i \epsilon\right)^{3 / 2}\left(F\left(-\frac{2 p}{4 q}+\left(\frac{p}{2 q}\right)^{2} \mathfrak{z}^{\prime}\right)-F\left(-\frac{2 p}{4 q}+\left(\frac{p}{2 q}\right)^{2} \epsilon^{\prime}\right)\right)+O(h) \\
& =2 e^{\frac{\pi}{4} i}\left(\frac{2 q}{p}+i \epsilon\right)^{3 / 2} S(4 q,-p) e^{-\frac{\pi}{4} i} \frac{1}{4|q|}\left|\frac{p}{2 q}\right| \sqrt{\mathfrak{z}^{\prime}}+O(h) \\
& =\left(\frac{p}{2|q|}\right)^{1 / 2} \frac{1}{p} S(4|q|,-\operatorname{sgn}(q) p) \sqrt{\mathfrak{z}^{\prime}}+O(h) .
\end{aligned}
$$

Now on letting $\epsilon \rightarrow 0$ we get the desired result.

As a corollary we note that:

Corollary 3.2. Let $x=\frac{q}{p}$ be of the form $\frac{2 A+1}{2 B+1}$, i.e. $p, q$ both being odd. Then

$$
R(2 A+1,2 B+1)=S(2 p, q)=0
$$

where $R$ is the coefficient in the forthcoming Eq. 19.

Proof.

$$
\begin{aligned}
S(2 p, q) & =e^{\frac{\pi}{4} i}\left(\frac{p}{2|q|}\right)^{1 / 2} S(4|q|, 2 \operatorname{sgn}(q) p) \\
& =e^{\frac{\pi}{2} i}\left(\frac{p}{2|q|}\right)^{1 / 2}\left(\frac{4|q|}{2|2 p|}\right)^{1 / 2} S(4 \cdot 2 p, 2 \operatorname{sgn}(q)|q|) \\
& =e^{\frac{\pi}{2} i} \frac{p}{|q|} \sqrt{\operatorname{sgn}(q)} S(2 p, \operatorname{sgn}(q)|q|) .
\end{aligned}
$$


We now conclude (17) by simply noting that $\operatorname{sgn}(q)|q|=q$.

Remark 2. The relation (9) leads to the so-called 'Landsberg-Schaar' identity (see [14], (5)) if we take $p$ and $q$ to be co-prime positive integers. This is

$$
\frac{1}{\sqrt{ } \bar{p}} \sum_{j=0}^{p-1} e^{2 \pi i j^{2} \frac{q}{p}}=\frac{e^{\frac{\pi}{4}} i}{\sqrt{2 q}} \sum_{j=0}^{2 q-1} e^{2 \pi i j^{2} \frac{p}{2 q}} .
$$

The following result will be required to complete the proof of the differentiability of Riemann's function.

\section{Lemma 3.3. For a natural number $p$,}

$$
S(p, q)=\varepsilon(p)\left(\frac{q}{p}\right) \sqrt{p}
$$

where $\left(\frac{q}{p}\right)$ indicates the Jacobi symbol and

$$
\varepsilon(p)= \begin{cases}1 & p \equiv 1 \bmod 4 \\ i & p \equiv 3 \bmod 4\end{cases}
$$

We are now ready to state a seemingly more general version of Theorem 2.1. This implies differentiability of Riemann's function at the rational point $\frac{2 A+1}{2 B+1}$ on putting $\mathfrak{z}=h+i \epsilon$ and $\epsilon \rightarrow+0$. We note that similar result is also obtained in ([3], Theorem 4.2).

\section{Corollary 3.4.}

$$
F\left(\frac{q}{p}+\mathfrak{z}\right)-F\left(\frac{q}{p}+i \epsilon\right)=R(p, q) \frac{e^{-\pi i / 4}}{p} \sqrt{\mathfrak{z}}-\frac{1}{2} h+O\left(\mathfrak{z}^{2}\right),
$$

where

$$
\begin{aligned}
& R(p, 2 q)=S(p, q)=\varepsilon(p)\left(\frac{q}{p}\right) \sqrt{p}, \\
& R(2 p, q)=S(4 p, q)=e^{\frac{\pi}{4} i} \sqrt{2 p}\left(\frac{-p}{q}\right) \\
& R(2 B+1,2 A+1)=0 .
\end{aligned}
$$

Proof. Only the case $R(2 p, q)$ needs to be considered (by Corollary 3.2). Now by (9) we have,

$$
\begin{aligned}
R(2 p, q)=S(4 p, q) & =e^{\frac{\pi}{4} i}\left(\frac{4 p}{2|q|}\right)^{1 / 2} S(4|q|,-4 \operatorname{sgn}(q) p) \\
& =e^{\frac{\pi}{4} i}\left(\frac{2 p}{|q|}\right)^{1 / 2} S(|q|,-\operatorname{sgn}(q) p) \\
& =e^{\frac{\pi}{4} i}\left(\frac{2 p}{|q|}\right)^{1 / 2} \sqrt{|q|} \varepsilon(|q|)\left(\frac{-\operatorname{sgn}(q) p}{|q|}\right) \\
& =e^{\frac{\pi}{4} i} \sqrt{2 p}\left(\frac{-p}{q}\right) .
\end{aligned}
$$


Remark 3. We make a historical remark on Riemann's function. [1] contains an almost complete list of references up to 1986. One addition is a correction to [17] in 1983. After this, the review of [6] contains an almost complete list after [1] except for [13] (esp. 619) and [18]. Among the papers listed in the review of [6], we mention [8] and [10] for consideration from the point of wavelets and [3] for self-similarity.

\section{Competing interests}

The authors declare that they have no competing interests.

\section{Acknowledgement}

The authors are indebted to the anonymous referee for his(her) enlightening comments which enabled us to make the paper more orderly (specially the 'Introduction')

The second author is supported in part by JSPS Grant in-aid for Scientific Research No. 17540050

To Professor Kálmán Györy on his seventy-fifth birthday with great respect and friendship.

\section{Author details}

${ }^{1}$ Harish-Chandra Research Institute, Chhatnag Road, Jhunsi, Allahabad 211019, India. ${ }^{2}$ Graduate School of Advanced Technology, Kinki University, lizuka, Fukuoka 820-8555, Japan. ${ }^{3}$ Department of Mathematics, Weinan Teachers College, Weinan, Shaanxi, 714000, People's Republic of China.

Received: 8 May 2015 Accepted: 7 July 2015

Published: 28 September 2015

\section{References}

1. Butzer, PL, Stark, EL: "Riemann's example" of a continuous nondifferentiable function in the light of two letters (1865) of Christoffel to Prym. Bull. Soc. Math. Belg. Sèr. A. 38, 45-73 (1986)

2. Cauchy, AL: Methode simple et nouvelle pour la determination complete des sommes alternee, formees avec les racines primitives des equattions binomes. J. Math. Pure Appl. (Liouvilee). 5, 154-183 (1840)

3. Duistermaat, JJ: Self-similarity of "Riemann's nondifferentiable function. Nieuw Arch. Wisk. 9, 303-337 (1991)

4. Gerver, J: The differentiability of the Riemann function at certain rational multiples of $\pi$. Amer. J. Math. 92, 33-55 (1970)

5. Gerver, J: More on the differentiability of the Riemann function. Amer. J. Math. 93, 33-41 (1970)

6. Gerver, J: On cubic lacunary Fourier series. Trans. Amer. Math. Soc. 355, 4297-4347 (2003)

7. Hardy, GH: Weierstrass non-differentiable functions. Trans. Amer. Math. Soc. 17, 301-325 (1916)

8. Holschneider, M, Tchamichian, P: Pointwise analysis of Riemann's nondifferentiable function. Invent. Math. 105, 157-275 (1991)

9. Itatsu, S: Differentiability of Riemann's function. Proc. Japan Acad. Ser. A Math. Sci. 57, 492-495 (1981)

10. Jaffard, S: The spectrum of sigularities of Riemann's function. Rev. Mat. Iberoamericana. 12, 441-460 (1996)

11. Kahane, JP: Lacunary Taylor and Fourier series. Trans. Amer. Math. Soc. 79, 199-213 (1964)

12. Kanemitsu, S, Tsukada, H: Contributions to the theory of zeta-functions-modular relation supremacy. World Scientific, London (2014)

13. Meyer, Y: Le traitment du signal et l'analyze mathématique. Ann. Inst. Fourier Grenoble. 50, 593-632 (1994)

14. Murty, R, Pacelli, A: Quadratic reciprocity via theta functions. Proc. Int. Conf.-Number Theory No. 1, 107-116 (2004)

15. Neuenschwander, E: Riemann's example of a continuous "non-differentiable" function. The Mathematical Intelligencer. 1(1), 40-44 (1978)

16. Segal, SL: Riemann's example of a continuous "non-differentiable" function continued. Math. Intell. 1(2), 81-82 (1978)

17. Smith, A: The differentiability of Riemann's function. Proc. Amer. Math. Soc. 34, 463-468 (1983)

18. Ulrich, P: Anmerkungen zum "Riemannschen Beispiel" einer stetigen, nicht differenzierbaren Funktion. Res. Math. 31, 245-265 (1997)

\section{Submit your manuscript to a SpringerOpen ${ }^{\odot}$ journal and benefit from:}

- Convenient online submission

- Rigorous peer review

- Immediate publication on acceptance

- Open access: articles freely available online

- High visibility within the field

- Retaining the copyright to your article

Submit your next manuscript at $>$ springeropen.com 